\title{
Electroweak couplings of the Higgs boson at a multi-TeV muon collider
}

\author{
Tao Han, ${ }^{1, *}$ Da Liu, ${ }^{2, \dagger}$ Ian Low, ${ }^{3,4, \hbar}$ and Xing Wang ${ }^{5, \S}$ \\ ${ }^{1}$ Department of Physics and Astronomy, University of Pittsburgh, Pittsburgh, Pennsylvania 15217, USA \\ ${ }^{2}$ Center for Quantum Mathematics and Physics (QMAP), University of California, \\ Davis, California 95616, USA \\ ${ }^{3}$ Department of Physics and Astronomy, Northwestern University, Evanston, Illinois 60208, USA \\ ${ }^{4}$ High Energy Physics Division, Argonne National Laboratory, Lemont, Illinois 60439, USA \\ ${ }^{5}$ Department of Physics, University of California-San Diego, La Jolla, California 92093, USA
}

(Received 13 October 2020; accepted 14 December 2020; published 6 January 2021)

\begin{abstract}
We estimate the expected precision at a multi- $\mathrm{TeV}$ muon collider for measuring the Higgs boson couplings with electroweak gauge bosons, $H V V$ and $H H V V\left(V=W^{ \pm}, Z\right)$, as well as the trilinear Higgs self-coupling $H H H$. At very high energies both single and double Higgs productions rely on the vectorboson fusion (VBF) topology. The outgoing remnant particles have a strong tendency to stay in the very forward region, leading to the configuration of the "inclusive process" and making it difficult to isolate $Z Z$ fusion events from the $W W$ fusion. In the single Higgs channel, we perform a maximum likelihood analysis on $H W W$ and $H Z Z$ couplings using two categories: the inclusive Higgs production and the 1-muon exclusive signal. In the double Higgs channel, we consider the inclusive production and study the interplay of the trilinear $\mathrm{HHH}$ and the quartic $\mathrm{VVHH}$ couplings, by utilizing kinematic information in the invariant mass spectrum. We find that at a center-of-mass energy of $10 \mathrm{TeV}(30 \mathrm{TeV})$ with an integrated luminosity of $10 \mathrm{ab}^{-1}\left(90 \mathrm{ab}^{-1}\right)$, one may reach a $95 \%$ confidence level sensitivity of $0.073 \%(0.023 \%)$ for $W W H$ coupling, $0.61 \%(0.21 \%)$ for $Z Z H$ coupling, $0.62 \%(0.20 \%)$ for $W W H H$ coupling, and $5.6 \%(2.0 \%)$ for $H H H$ coupling. For dim- 6 operators contributing to the processes, these sensitivities could probe the new physics scale $\Lambda$ in the order of $1-10(2-20) \mathrm{TeV}$ at a $10 \mathrm{TeV}(30 \mathrm{TeV})$ muon collider.
\end{abstract}

DOI: 10.1103/PhysRevD.103.013002

\section{INTRODUCTION}

The discovery of the Higgs boson at the CERN Large Hadron Collider (LHC) opens a new avenue in particle physics. On the one hand, the existence of the Higgs boson completes the particle spectrum in the Standard Model (SM) and provides a self-consistent mechanism in quantum field theory for mass generation of elementary particles. On the other hand, the SM does not address the underlying mechanism for the electroweak symmetry breaking (EWSB) and thus fails to understand the stability of the weak scale with respect to the Planck scale. In order to gain further insight for those fundamental questions, it is of high priority to study the Higgs boson properties to high

\footnotetext{
*than@pitt.edu

daeliu@ucdavis.edu

*ilow@northwestern.edu

§iw006@physics.ucsd.edu
}

Published by the American Physical Society under the terms of the Creative Commons Attribution 4.0 International license. Further distribution of this work must maintain attribution to the author(s) and the published article's title, journal citation, and DOI. Funded by SCOAP . precision in the hope to identify hints for new physics beyond the SM.

In the SM, the Higgs sector is constructed from a complex scalar doublet $\Phi$. After the EWSB, the neutral real component is the Higgs boson excitation $H$ and the other three degrees of freedom become the longitudinal components of the massive gauge bosons. As such, studying the Higgs-gauge boson couplings would be the most direct probe to the underlying mechanism of the electroweak symmetry breaking. After the EWSB, the Higgs sector can be parametrized as

$$
\begin{aligned}
\mathcal{L} \supset & \left(M_{W}^{2} W_{\mu}^{+} W^{-\mu}+\frac{1}{2} M_{Z}^{2} Z_{\mu} Z^{\mu}\right)\left(\kappa_{V} \frac{2 H}{v}+\kappa_{V_{2}} \frac{H^{2}}{v^{2}}\right) \\
& -\frac{m_{H}^{2}}{2 v}\left(\kappa_{3} H^{3}+\frac{1}{4 v} \kappa_{4} H^{4}\right),
\end{aligned}
$$

where $v=246 \mathrm{GeV}$ is the vacuum expectation value of the Higgs field and $\kappa_{i}=1$ for the SM couplings at tree level. This " $\kappa$-scheme" is a convenient phenomenological parametrization of deviations from the SM expectations, which is suitable for the exploratory nature of the present study. Here it is made implicit that $\kappa_{V}=\kappa_{W}=\kappa_{Z}$. This is the prediction of the tree-level custodial SU(2) invariance [1], 
which is an accidental symmetry of the SM. This has been verified to a good accuracy by precision EW measurements [2]. Nevertheless, in our fit we wish to be more general and will not be assuming a correlated $\kappa_{W}$ and $\kappa_{Z}$.

A fully consistent and theoretically-sound framework would utilize effective field theories (EFT), by augmenting the SM Lagrangian with higher dimensional operators from integrating out the heavier states [3]. While a systematic account for the effects of the higher dimensional operators is much more involved and beyond the scope of the current work, we would like to consider the following two operators for the purpose of illustration $[4,5]$

$\mathcal{O}_{H}=\frac{c_{H}}{2 \Lambda^{2}} \partial_{\mu}\left(\Phi^{\dagger} \Phi\right) \partial^{\mu}\left(\Phi^{\dagger} \Phi\right), \quad \mathcal{O}_{6}=-\frac{c_{6} \lambda}{\Lambda^{2}}\left(\Phi^{\dagger} \Phi\right)^{3}$,

where $\Lambda$ is the cutoff scale where new physics sets in, and $\lambda$ is the quartic coupling parameter in front of $\left(H^{\dagger} H\right)^{2}$ term in the SM Higgs potential. At the dimension-six level these are the two operators that are most relevant for our study. An additional operator, $\Phi^{\dagger} \Phi\left(D_{\mu} \Phi\right)^{\dagger}\left(D^{\mu} \Phi\right)$, can be removed by a suitable field-redefinition [5]. The resulting shifts $\Delta \kappa_{i} \equiv \kappa_{i}-1$ in Eq. (1) are $^{1}$

$$
\begin{aligned}
& \Delta \kappa_{V}=-\frac{c_{H}}{2} \frac{v^{2}}{\Lambda^{2}}, \quad \Delta \kappa_{V 2}=-2 c_{H} \frac{v^{2}}{\Lambda^{2}}, \\
& \Delta \kappa_{3} \approx-\frac{3 c_{H}}{2} \frac{v^{2}}{\Lambda^{2}}+c_{6} \frac{v^{2}}{\Lambda^{2}}, \quad \Delta \kappa_{4} \approx-\frac{25}{9} c_{H} \frac{v^{2}}{\Lambda^{2}}+6 c_{6} \frac{v^{2}}{\Lambda^{2}},
\end{aligned}
$$

We see that deviations in the $V V H$ and $V V H H(V=$ $\left.W^{ \pm}, Z\right)$ couplings are correlated and controlled by the same operator $\mathcal{O}_{H}$. However, the precision we are expecting is high and could potentially be sensitive to effects of dimension- 8 operators, in which case the correlation may be modified. On the other hand, the Higgs trilinear selfcoupling $\kappa_{3}$ is among the most important interactions to be tested in the Higgs sector-it governs the shape of the Higgs potential and, consequently, the nature of the electroweak symmetry breaking. In addition, $\kappa_{3}$ controls the strength of the electroweak phase transition, which is important for understanding the cosmological evolution of the early universe as well as the origin of the observed matter-antimatter asymmetry in the current unverse [7-9]. Precise measurements of these couplings will provide insights on how nature works at the shortest distance scale ever probed by mankind. Needless to say, should deviations from the SM predictions be observed, it would completely revolutionize our understanding of the physical laws of nature.

\footnotetext{
${ }^{1}$ Interestingly, in most cases there is a positivity constraint on $c_{H}>0$, thereby reducing the $V V H$ and $V V H H$ coupling strengths [6].
}

With the great success of the LHC program, we have achieved the measurement of the $V V H$ to $\mathcal{O}(5 \%)$ accuracy $[10,11]$, which will be further improved by roughly a factor of two with the high-luminosity LHC upgrade [12]. In $e^{+} e^{-}$collisions at the International Linear Collider (ILC) $[13,14]$, the proposed Higgs factories [15-17] and the CLIC [18,19], subpercent level accuracies for $W W H$ of $\mathcal{O}(0.6 \%-1.2 \%)$ and $\mathrm{ZZH}$ of $\mathcal{O}(0.2 \%-0.5 \%)$ could be achievable. However, the trilinear $\mathrm{HHH}$ and quartic $V V H H$ couplings are still difficult to measure to an informative level without a very high energy collider $[20,21]$. At a $100 \mathrm{TeV}$ hadron collider such as the SPPC or $\mathrm{FCC}_{h h}$, the trilinear Higgs self-coupling could be measured with $\mathcal{O}(5 \%)$ uncertainty [22,23] and the $V V H H$ coupling could be measured with $\mathcal{O}(1 \%)$ uncertainty [24]. Recently, an attempt was made to determine the quartic Higgs self-coupling at a high-energy muon collider [25]. In the EFT language, the precision to which one could measure the Higgs couplings can be translated into constraints on the scale suppressing dimension- 6 operators, which is indicative of the scale where new physics becomes important. A figure of merit is when $\Lambda \sim 1 \mathrm{TeV}$ which, generally speaking, would induce a corresponding deviation in the Higgs couplings of the order [26]

$$
\mathcal{O}\left(\frac{v^{2}}{\Lambda^{2}}\right) \sim \mathcal{O}(5 \%) \text { for } \Lambda \sim 1 \mathrm{TeV} .
$$

Therefore, in order to probe new physics scale above $1 \mathrm{TeV}$, it is important to be able to reach a precision level of 5\% or less. In addition, in a lepton collider a truly modelindependent determination of the trilinear $\mathrm{HHH}$ coupling requires simultaneously measuring the 4-point $V V H H$ coupling, which is difficult to access at low energies and without sufficiently high statistics.

Recently, there has been a renewed interest to consider a muon collider with a very high center-of-mass (CM) energy in the tens of $\mathrm{TeV}$ [27-32]. While the previous discussions for a muon collider were focused on a Higgs factory operating at the SM Higgs resonance [33,34], a collider operating at a multi- $\mathrm{TeV}$ regime would certainly lead us to a new territory at the energy frontier. Such a multi-TeV muon collider offers a unique opportunity to probe the electroweak couplings of the Higgs boson, including $\mathrm{VVH}, \mathrm{HHH}$ and $V V H H$ couplings. The possible $\mathrm{CM}$ energy under discussion ranges from $3 \mathrm{TeV}$ to $30 \mathrm{TeV}$, with a representative benchmark target at $10 \mathrm{TeV}$ or higher. Very high luminosities are also envisioned, with the scaling relation as [27]

$$
\text { Lumi }>\frac{5 \text { years }}{\text { time }}\left(\frac{\sqrt{s}}{10 \mathrm{TeV}}\right)^{2} 2 \times 10^{35} \mathrm{~cm}^{-2} \mathrm{~s}^{-1} \text {. }
$$

This will yield to an integrated luminosity of $\mathcal{O}(10) \mathrm{ab}^{-1}$ at $\sqrt{s}=10 \mathrm{TeV}$ and $\mathcal{O}(90) \mathrm{ab}^{-1}$ at $\sqrt{s}=30 \mathrm{TeV}$, which 
would take us to a remarkable new energy frontier, and offer great potential to study the Higgs boson, and the Nature in general at an unprecedented short-distance scales. In this paper, we would like to explore the Higgs physics and examine the accuracies for the electroweak couplings of the Higgs boson at the future high-energy muon collider.

The rest of the paper is organized as follows. We first present the Higgs boson production rates via various production mechanisms at a high-energy muon collider in Sec. II. We then evaluate the statistical accuracy achievable to determine the $H V V$ couplings in Sec. III. Foremost, we show the improvement for the precision measurement on the triple Higgs boson coupling as well as the $V V H H$ coupling in Sec. IV. We summarize our results and conclude in Sec. V.

\section{HIGGS BOSON PRODUCTION AT A HIGH- ENERGY MUON COLLIDER}

The Higgs boson couples predominantly to heavier particles. The production of a Higgs boson thus involves other heavy particles in the SM. At high energies, gauge bosons will copiously radiate off the colliding beams. Therefore, the vector boson fusion (VBF) mechanism are the dominant source for the Higgs boson production at a high-energy muon collider [30,31]. The production processes involving the Higgs boson at a high-energy muon collider include

$$
\mu^{+} \mu^{-} \stackrel{\mathrm{VBF}}{\rightarrow} H, \quad Z H, \quad H H \quad \text { and } \quad t \bar{t} H,
$$

which are all dominantly from the VBF processes. We list the production cross sections in Table I for those Higgs production processes with a few representative benchmark energy choices. Cross sections are computed using the package MadGraph [35]. Recently it has been advocated that, in high energy collisions, it may be appropriate to adopt the approach of electroweak parton distribution functions (EW PDF) [31] to resum the potentially large collinear

TABLE I. SM Higgs boson production cross sections in units of $\mathrm{fb}$ at a muon collider for various energies. For comparison, the SM background processes of $Z$ and $Z Z$ production are also shown.

\begin{tabular}{lccccc}
\hline \hline$\sqrt{s}(\mathrm{TeV})$ & 3 & 6 & 10 & 14 & 30 \\
\cline { 2 - 6 } benchmark lumi $\left(\mathrm{ab}^{-1}\right)$ & 1 & 4 & 10 & 20 & 90 \\
\hline$\sigma(\mathrm{fb}): W W \rightarrow H$ & 490 & 700 & 830 & 950 & 1200 \\
$Z Z \rightarrow H$ & 51 & 72 & 89 & 96 & 120 \\
$W W \rightarrow H H$ & 0.80 & 1.8 & 3.2 & 4.3 & 6.7 \\
$Z Z \rightarrow H H$ & 0.11 & 0.24 & 0.43 & 0.57 & 0.91 \\
$W W \rightarrow Z H$ & 9.5 & 22 & 33 & 42 & 67 \\
$W W \rightarrow t \bar{t} H$ & 0.012 & 0.046 & 0.090 & 0.14 & 0.28 \\
$W W \rightarrow Z$ & 2200 & 3100 & 3600 & 4200 & 5200 \\
$W W \rightarrow Z Z$ & 57 & 130 & 200 & 260 & 420 \\
\hline \hline
\end{tabular}

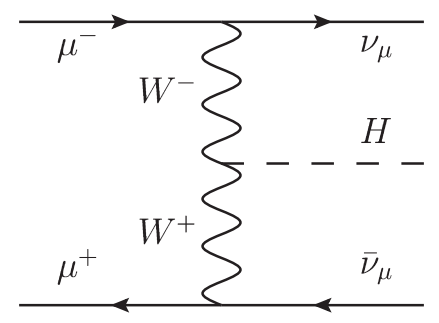

FIG. 1. VBF production of a single Higgs boson at a high energy muon collider via $W W$ fusion. For $Z Z$ fusion, replace the $W$ propagator by the $Z$ propagator and the outgoing neutrinos by muons.

logarithms at high scales. For the processes under consideration, the difference is insignificant since the single Higgs production is set by a low scale $m_{H}$, while the Higgs pair production $H H$ is dominated by the longitudinal gauge boson fusion $\left(W_{L} W_{L}\right)$, that has no scale dependence at the leading order.

We will examine the precision measurements of the Higgs boson couplings via the production processes as depicted in Figs. 1 and 2. For instance, at a $10 \mathrm{TeV}$ muon collider with an integrated luminosity of $10 \mathrm{ab}^{-1}$, we may expect the production of about $10^{7}$ Higgs bosons and $3.6 \times$ $10^{4}$ Higgs pairs. For comparison, we have also included in Table I the SM irreducible backgrounds $\mu^{+} \mu^{-} \stackrel{\mathrm{VBF}}{\rightarrow} Z, Z Z$, which are also largely from the VBF mechanism, in Table I. Although the background rates are larger than the signals by a factor of 4 (55) for the $H(H H)$ process, they populate different kinematical regions from the signals and can be reduced by appropriate kinematic cuts.

\section{VVH COUPLINGS}

At high energy lepton colliders, the cross section for single $H$ production via the Higgs-strahlung $\mu^{+} \mu^{-} \rightarrow Z H$ falls as $1 / s$. The high statistics channels for measurements of $V V H$ couplings rely on the $W W$ and $Z Z$ fusion via the VBF topology:

$$
\begin{aligned}
& \mu^{+} \mu^{-} \rightarrow \nu_{\mu} \bar{\nu}_{\mu} H \quad(W W \text { fusion }), \\
& \mu^{+} \mu^{-} \rightarrow \mu^{+} \mu^{-} H \quad(Z Z \text { fusion }) .
\end{aligned}
$$

See Fig. 1 for a representative Feynman diagram. It would be desirable to separate these two classes of events by tagging the outgoing muons and achieve independent measurements on $W W H$ and $Z Z H$ couplings. However, for the VBF topology, the outgoing muons have a tendency to stay in the forward region due to the $t$-channel propagator shown in Fig. 1. Although the transverse momentum of the outgoing muons is sizable and governed by the propagator mass $p_{T}^{\mu} \sim M_{Z}$, at very high energies the muons are all extremely forward with a scattering angle typically $\theta_{\mu} \approx M_{Z} / E_{\mu}$. In Fig. 3(a), we show the angular 


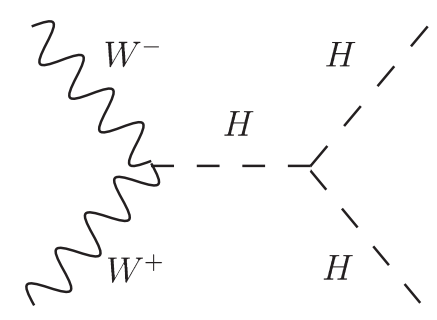

(a)

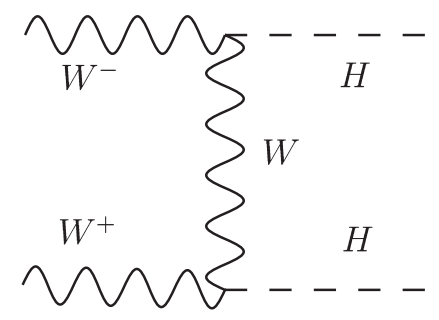

(b)

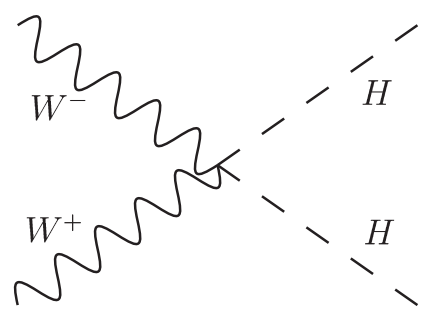

(c)

FIG. 2. Double Higgs production at a high energy muon collider via $W W$ fusion. The production goes through the VBF topology, as in Fig. 1.

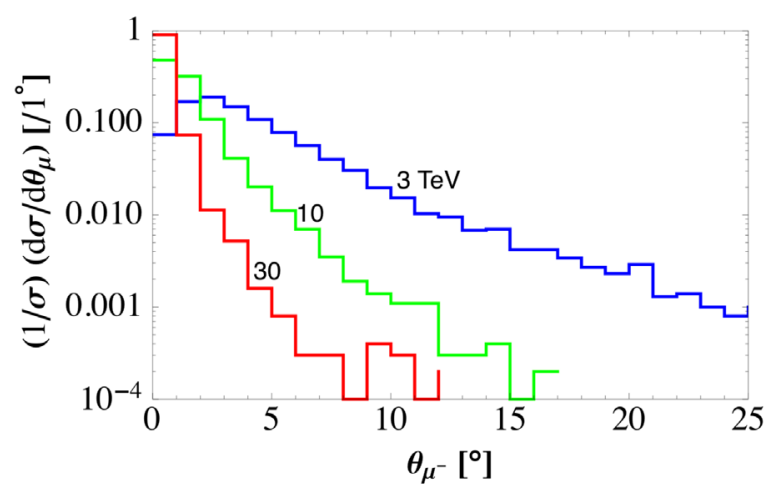

(a)

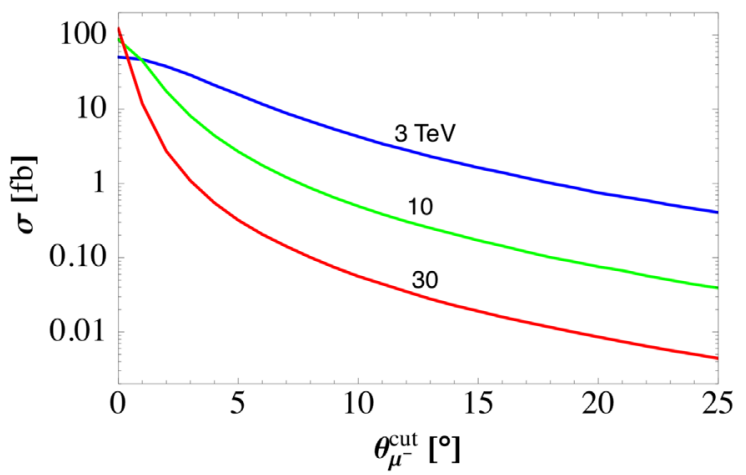

(b)

FIG. 3. $\mu^{+} \mu^{-} \rightarrow \mu^{+} \mu^{-} H$ via $Z Z$ fusion with $\sqrt{s}=3,10$ and $30 \mathrm{TeV}$ for (a) angular distribution $\theta_{\mu^{-}}$, and (b) total cross section versus an angular cut $\theta_{\mu^{-}}^{\text {cut }}$.

distributions of the outgoing muons at $\sqrt{s}=3,10,30 \mathrm{TeV}$. One can see that, for example, the scattering angle for a muon is peaked near $\theta_{\mu} \sim 0.02 \approx 1.2^{\circ}$ at $10 \mathrm{TeV}$. These very forward muons would most likely escape the detection in a detector at a few degrees away from colliding beams. This feature makes it increasingly difficult to distinguish the processes of the neutral currents ( $Z Z$ fusion [36]) from the charged currents ( $W W$ fusion) at higher energies. Therefore, separating these two classes of events would require the capability of detecting very energetic muons in the forward region and dedicated advanced detector design would be needed [37]. Without this, we would have to focus on the "inclusiveness," a dominant behavior of the collinear splitting physics recently emphasized in Ref. [31]. As a consequence, we will consider two classes of events for $\mathrm{VBF}$ production of single $H$ :

(i) Inclusive channel: events from $W W$ fusion and from $Z Z$ fusion without detecting muons;

(ii) Exclusive $1 \mu$ channel: events from $Z Z$ fusion with at least one muon detected.

The inclusive channel is populated predominantly by events from the $W W$ fusion, but also contains events from $Z Z$ fusion when the outgoing muons go down the beam pipe and escape detection. However, as seen from Table I, $Z Z$-fusion cross section is roughly $10 \%$ of the $W W$ fusion cross section, and thus a small contamination for the $W W H$ measurement. The $1 \mu$ channel, on the other hand, comes from the $Z Z$ fusion and is uniquely sensitive to the $Z Z H$ coupling, although it suffers from poor selection efficiency after requiring a muon identification. In Fig. 3(b), we illustrate the fiducial cross section after the angular acceptance cut $\theta_{\mu^{-}}^{\text {cut }}$. At a fixed angular acceptance, the cross section falls as $\sigma \sim 1 / E_{\mu}^{2}$.

\section{A. Inclusive channel}

Processes contributing to the inclusive channel are shown in Eqs. (7) and (8). We focus on the leading decay channel $H \rightarrow b \bar{b}$. The Higgs boson signal will be $b \bar{b}$ pair near the Higgs mass $m_{H}$ plus large missing energy, resulting from the missing neutrinos and the undetected muons. We impose the basic acceptance cuts on the $b$ jets

$$
p_{T}(b)>30 \mathrm{GeV}, \quad 10^{\circ}<\theta_{b}<170^{\circ},
$$

where $\theta_{b}$ is the polar angle of the $b(\bar{b})$ jet in the lab frame. The irreducible backgrounds, $\mu^{+} \mu^{-} \rightarrow \nu_{\mu} \bar{\nu}_{\mu} Z$, from either $W W$ fusion shown in Table I or $\mu^{+} \mu^{-} \rightarrow Z Z \rightarrow \nu_{\mu} \bar{\nu}_{\mu} Z$, which can be readily removed due to the on-shell $Z$ decay $Z \rightarrow \nu_{\mu} \bar{\nu}_{\mu}$, by a "recoil mass" cut 


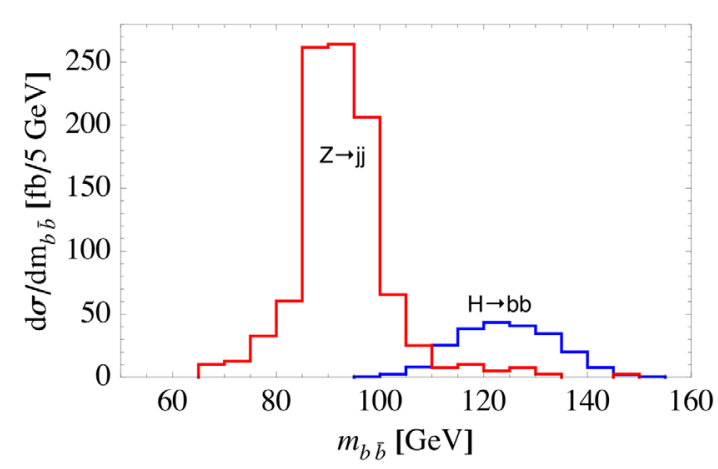

(a)

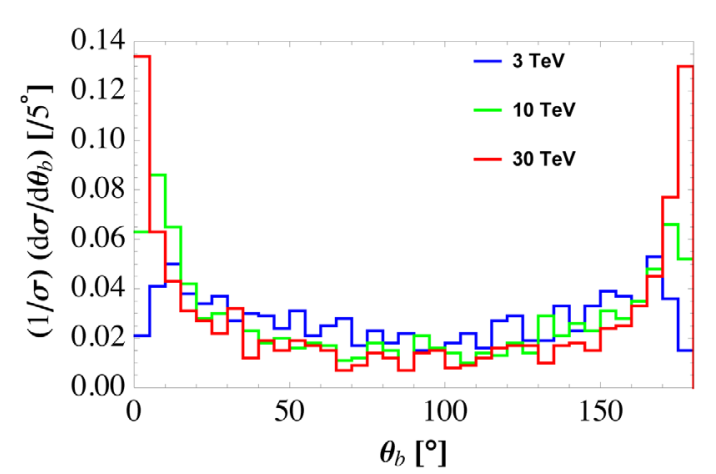

(b)

FIG. 4. (a) Invariant mass distribution for the Higgs boson and $Z$ boson at $\sqrt{s}=10 \mathrm{TeV}$ with an energy resolution $10 \%$, and (b) the $b$-quark angular distribution $\theta_{b}$ in the lab frame for $\sqrt{s}=3,10,30 \mathrm{TeV}$.

$$
M_{\text {recoil }}=\sqrt{\left(p_{\mu^{+}}+p_{\mu^{+}}-p_{H}\right)^{2}}>200 \mathrm{GeV} .
$$

The key aspect to identify the Higgs signal lies in the resolution to effectively select the $b \bar{b}$ at the resonant $m_{H}$. In Fig. 4(a) we plot the invariant mass distribution for the $H$ signal for $\sqrt{s}=10 \mathrm{TeV}$, after the acceptance cuts and assuming a jet energy resolution of

$$
\Delta E / E=10 \%
$$

For comparison, we have also shown in the same plot the distribution from the $Z$ background. Here we have included all quarks flavors $b, c, s, d, u$. If we demanded a $b$-tagging for our signal selection, we would be able to reduce the $Z \rightarrow j j$ background by about $23 \%$. However, we do not find the $b$-tagging necessary due to the highly efficient kinematical constraint on $m_{b \bar{b}}$. In estimating the statistical accuracy for the coupling measurement, we impose the a mass cut

$$
m_{b \bar{b}}=m_{H} \pm 15 \mathrm{GeV}
$$

With those cuts, the $Z$ background is essentially removed and we retain the majority of the signal. The event selection

TABLE II. Selection efficiencies and the estimated cross sections after selection cuts for the inclusive channel, exclusive $1 \mu$ channel, as well as the inclusive $H H$ channel.

\begin{tabular}{lccccc}
\hline \hline$\sqrt{s}(\mathrm{TeV})$ & 3 & 6 & 10 & 14 & 30 \\
\hline$W W \rightarrow H: \epsilon_{\text {in }}(\%)$ & 54 & 46 & 42 & 39 & 32 \\
$Z Z \rightarrow H: \epsilon_{\text {in }}(\%)$ & 57 & 49 & 44 & 41 & 35 \\
Cross section $\sigma_{\text {in }}(\mathrm{fb})$ & 170 & 200 & 220 & 240 & 240 \\
$Z Z \rightarrow H: \epsilon_{1 \mu}(\%)$ & 11 & 2.7 & 0.84 & 0.37 & 0.071 \\
Cross section $\sigma_{1 \mu}(\mathrm{fb})$ & 3.1 & 1.1 & 0.43 & 0.20 & 0.050 \\
$V V \rightarrow H H: \epsilon_{h h}(\%)$ & 27 & 18 & 13 & 11 & 7.2 \\
Cross section $\sigma_{h h}(\mathrm{ab})$ & 81 & 140 & 150 & 170 & 200 \\
\hline \hline
\end{tabular}

efficiencies $\left(\epsilon_{\text {in }}\right)$ and the resulting cross sections at different collider energies are summarized in Table II in the top rows.

It is worth noting that, at higher $\mathrm{CM}$ energies, the $b$ jets have increasingly small polar angles in the Lab frame and become more forward. The angular distributions for various energies are shown in the right panel of Fig. 4, where we see the majority of $b$ jets have $\theta_{b}<10^{\circ}$ at $\sqrt{s}=30 \mathrm{TeV}$. This is the reason for the worsening selection efficiencies in Table II as we go to higher CM energies. Obviously, extending the detector angular coverage would significantly increase the signal acceptance. If the angular cut on $\theta_{b}$ in Eq. (9) is tightened up to $20^{\circ}-160^{\circ}$ instead, the signal reconstruction efficiency will be scaled down by about $10 \%$.

The total cross section in the inclusive channel can be written, at the leading order, as

$$
\sigma_{\text {in }}=\left(1+\Delta \kappa_{W}\right)^{2} \sigma_{W}^{\mathrm{SM}}+\left(1+\Delta \kappa_{Z}\right)^{2} \sigma_{Z}^{\mathrm{SM}}
$$

where $\sigma_{W}^{\mathrm{SM}}$ and $\sigma_{Z}^{\mathrm{SM}}$ are the SM cross sections for the $W W / Z Z$ fusion processes. In cases where $\Delta \kappa_{W / Z} \ll 1$, the linear terms dominate which, in the EFT language, is equivalent to keeping only the interference term from the dim- 6 operators. We do not make such an assumption in the $\kappa$-scheme adopted in this work.

In this subsection we will vary $\kappa_{W}$ and $\kappa_{Z}$ one at a time, and consider a simultaneous fit to both parameters later in this section. The 95\% confidence level (C.L.) sensitivities

TABLE III. The $95 \%$ C.L. in $\Delta \kappa_{W / Z}$ in the inclusive channel by varying one coupling at a time, as well as for $\Delta \kappa_{Z}$ from the exclusive $1 \mu$ process.

\begin{tabular}{lccccc}
\hline \hline$\sqrt{s}(\mathrm{TeV})$ & 3 & 6 & 10 & 14 & 30 \\
\cline { 2 - 6 } Benchmark lumi $\left(\mathrm{ab}^{-1}\right)$ & 1 & 4 & 10 & 20 & 90 \\
\hline$\left(\Delta \kappa_{W}\right)_{\text {in }}$ & $0.26 \%$ & $0.12 \%$ & $0.073 \%$ & $0.050 \%$ & $0.023 \%$ \\
$\left(\Delta \kappa_{Z}\right)_{\text {in }}$ & $2.4 \%$ & $1.1 \%$ & $0.65 \%$ & $0.46 \%$ & $0.20 \%$ \\
$\left(\Delta \kappa_{Z}\right)_{1 \mu}$ & $1.7 \%$ & $1.5 \%$ & $1.5 \%$ & $1.5 \%$ & $1.5 \%$ \\
\hline \hline
\end{tabular}




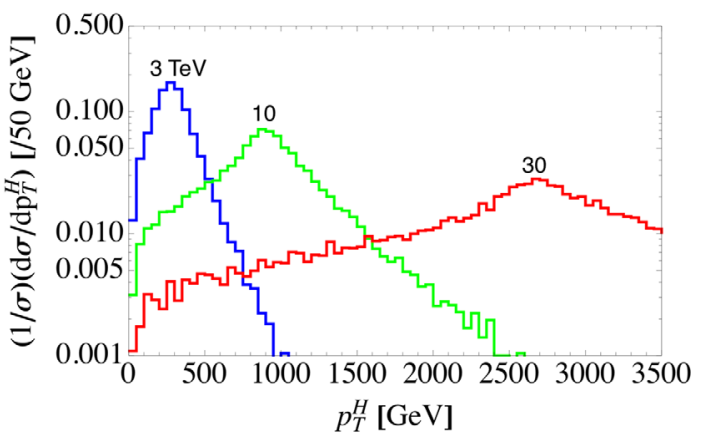

(a)

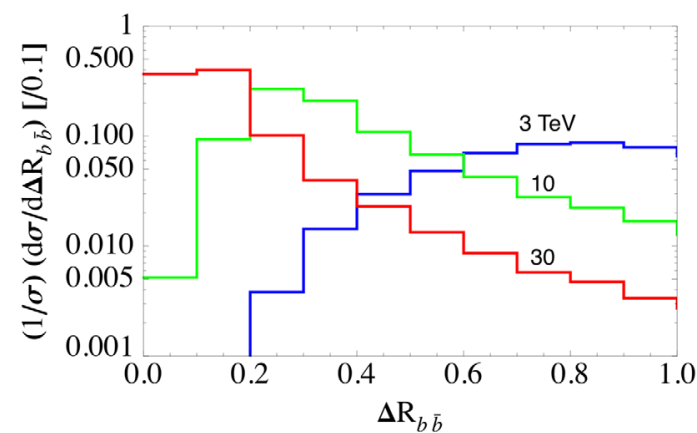

(b)

FIG. 5. (a) $p_{T}^{H}$ distribution of the Higgs boson in $1 \mu$ channel (b) Separation of the $b$ jets from $H \rightarrow b \bar{b}$.

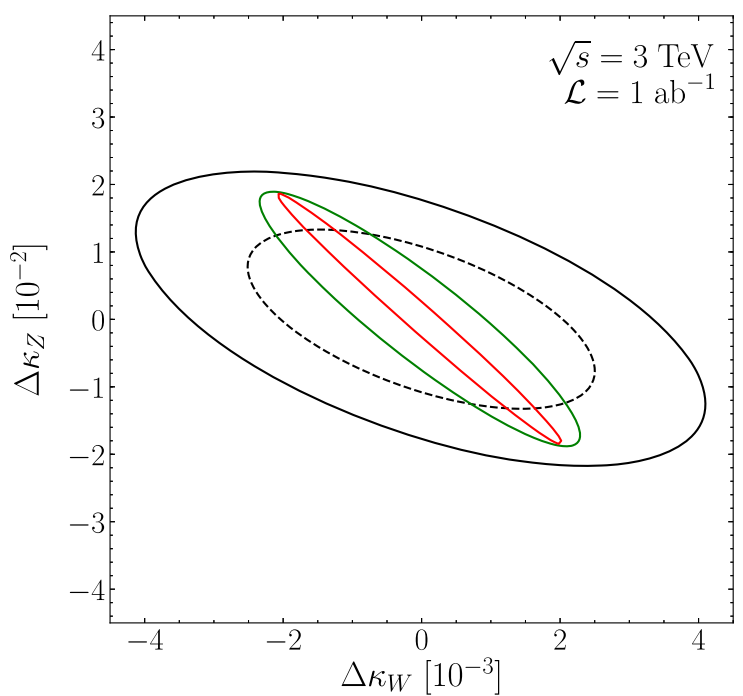

(a)

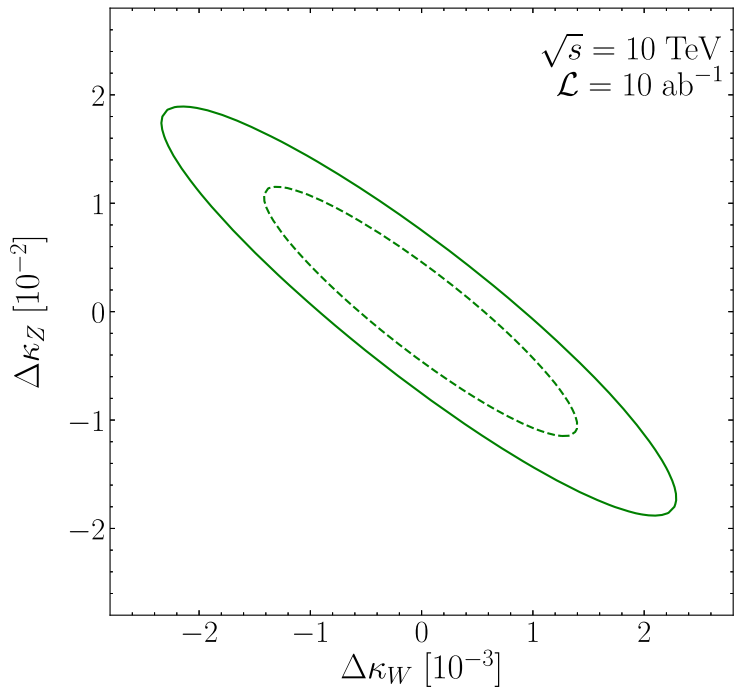

(c)

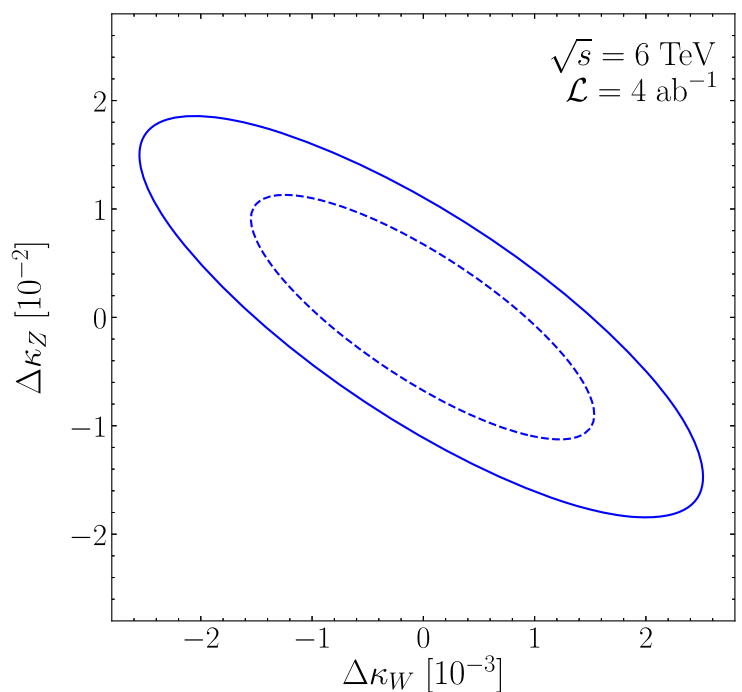

(b)

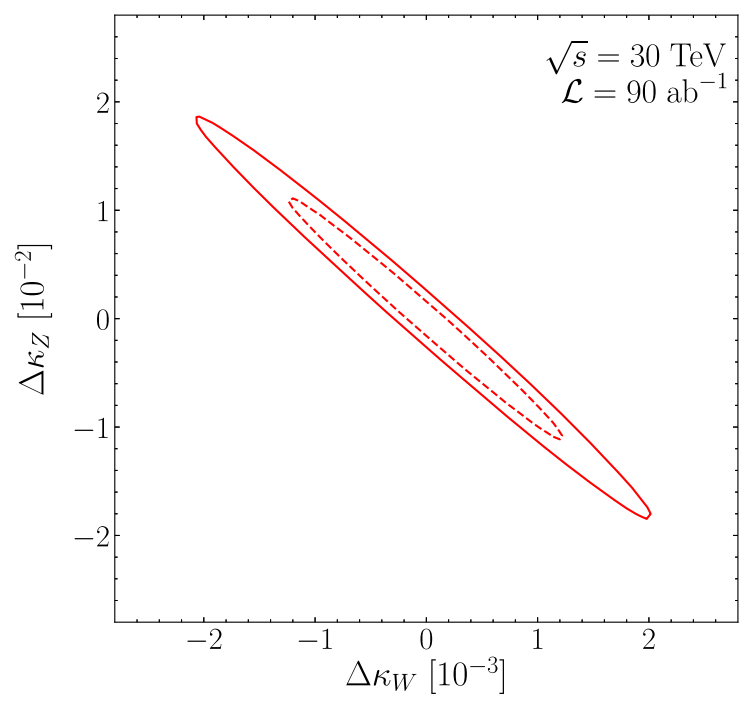

(d)

FIG. 6. Correlated bounds with $95 \%$ C.L. (solid) and $68 \%$ C.L. (dashed) in the $\Delta \kappa_{W}-\Delta \kappa_{Z}$ plane for $\sqrt{s}=3,6,10,30$ TeV, respectively. In (a), inner ellipses (solid) include the 95\% C.L. results for $10 \mathrm{TeV}$ and $30 \mathrm{TeV}$ for comparison. 
in the relative errors $\Delta \kappa_{W / Z}$ are shown in Table III. The achievable accuracies are impressive for the $\Delta \kappa_{W}$, comparing with the anticipated best results $\Delta \kappa_{W} \sim 0.1 \%$ from the ILC/CLIC, while for the $\Delta \kappa_{Z}$, it is comparable with $\Delta \kappa_{Z} \sim$ $0.13 \%$ from the expectations at the Higgs factories $[15,17]$.

\section{B. Exclusive $1 \mu$ channel}

The leading process contributing to the exclusive $1 \mu$ channel is $Z Z$ fusion in Eq. (8), whose rate is shown in Table I. Again, with the same decay mode, the Higgs boson signal will be $b \bar{b}$ pair near the Higgs mass $m_{H}$ plus $\mu^{+} \mu^{-}$in the forward-backward regions. The leading background is $\mu^{+} \mu^{-} \rightarrow Z Z \rightarrow \mu^{+} \mu^{-} Z$ with $Z \rightarrow b \bar{b}$. There is no $W W$ fusion analogue for this channel. We adopt the same basic cuts as in Eqs. (9), (10) and (12). The background is highly suppressed. In addition, we require the presence of at least one muon to be in

$$
10^{\circ}<\theta_{\mu^{ \pm}}<170^{\circ} \text {. }
$$

This turns out to be very costly to the signal, since the majority of the muons have $\theta_{\mu}<10^{\circ}$, as already seen in Fig. 3. As such, the signal reconstruction efficiencies for this channel are very low and are shown in Table II, together with the predicted cross sections in the middle rows. With the high luminosity expected, the $95 \%$ C.L. on the coupling measurements is shown also in Table III for the exclusive $1 \mu$ channel. Although the result at a $3 \mathrm{TeV}$ collider is comparable to that from the inclusive channel, at higher energies the estimated precision is worse than the inclusive channel despite the higher energies and more luminosities. This is mainly due to the significantly reduced number of events from the tagging requirement for a forward-backward muon.

It is important to note another significant consequence of requiring one muon in the range of $10^{\circ}<\theta_{\mu^{ \pm}}<170^{\circ}$. For highly energetic muons, this large scattering angle leads to a high transverse momentum $p_{T}^{\mu}>0.17 E_{\mu}$ and, consequently, induces a strong recoil in the Higgs boson produced in the final state. In Fig. 5 we show the $p_{T}$ distribution of the Higgs boson in (a) for the $1 \mu$ channel as well as $R_{b b}$ in (b), the separation of the $b$-jets from $H \rightarrow b \bar{b}$. In particular, at $\sqrt{s}=30 \mathrm{TeV}$, the Higgs boson tend to have a large $p_{T}$, in the order of $2.5 \mathrm{TeV}$, and the resulting decay is boosted with $R_{b b} \sim 0.2$. Care needs to be taken when reconstructing such boosted events.

\section{Two-parameter likelihood fit of $\kappa_{W}$ and $\kappa_{Z}$}

In this subsection we perform a two-bin likelihood fit of $\kappa_{W}$ and $\kappa_{Z}$ making use of the inclusive and exclusive $1 \mu$ channels. We construct a Poisson log-likelihood function

$$
\mathrm{LL}=\ln \frac{e^{-N\left(\Delta \kappa_{W}, \Delta \kappa_{Z}\right)}\left[N\left(\Delta \kappa_{W}, \Delta \kappa_{Z}\right)\right]^{N_{\mathrm{SM}}}}{N_{\mathrm{SM}} !}
$$

where the numbers of events are

$$
\begin{aligned}
N\left(\Delta \kappa_{W}, \Delta \kappa_{Z}\right) & =\sigma\left(\Delta \kappa_{W}, \Delta \kappa_{Z}\right) \mathcal{L}_{\text {lumi }} \\
N_{\mathrm{SM}} & =\sigma\left(\Delta \kappa_{W}=0, \Delta \kappa_{Z}=0\right) \mathcal{L}_{\text {lumi }},
\end{aligned}
$$

and $\mathcal{L}_{\text {lumi }}$ is the integrated luminosity. We compute such likelihood function for each channel and a global likelihood as the product of the individual ones. Then we compute the $68 \%$ and $95 \%$ C.L. regions on the $\Delta \kappa_{W}-\Delta \kappa_{Z}$ plane, corresponding to $\mathrm{LL}=\mathrm{LL}_{\max }-1.15$ and $\mathrm{LL}=\mathrm{LL}_{\max }-$ 3.10 , respectively. The resulting contours are shown in Fig. 6. As expected, the precision for $\Delta \kappa_{W}$ is better than $\Delta \kappa_{Z}$ by about an order of magnitude at high energies. The projection of the ellipses onto the $\Delta \kappa_{W}$-axis in Fig. 6 gives the uncertainty marginalized over $\Delta \kappa_{Z}$, and vice versa. The resulting errors are larger than those in the single parameter fit, which varies one parameter at a time and assumes SM values for the rest.

\section{HHH AND WWHH COUPLINGS}

Pair production of the Higgs boson provides a direct measurement on the trilinear $\mathrm{HHH}$ and quartic $\mathrm{VVHH}$ couplings. The main advantage of a high-energy collider, with $\sqrt{s} \gg 2 m_{H}$, lies in the capability to copiously produce Higgs boson pairs. At a high-energy muon collider, as shown in Sec. II, one would expect about 36,000 $(680,000)$ $H H$ at $10 \mathrm{TeV}(30 \mathrm{TeV})$. To probe the Higgs self-coupling, we utilize the VBF mechanism for the inclusive double Higgs production

$$
\mu^{+} \mu^{-} \stackrel{\mathrm{VBF}}{\rightarrow} H H+X
$$

where $X=\nu \bar{\nu}$ for $W W$ fusion and $\mu^{+} \mu^{-}$for the $Z Z$ fusion. As can be seen from the Feynman diagrams in Fig. 2, the $H H$ production involves three couplings: $\kappa_{W}, \kappa_{3}$ and $\kappa_{W 2}$. Since $\kappa_{W}$ can be measured very precisely from the single Higgs production, as shown in Sec. III, we will assume in the current section that $\kappa_{W}=1$ as in the SM and study the interplay of $\kappa_{3}$ and $\kappa_{W 2}$ in the $H H$ production. As discussed in Sec. III, the outgoing remnant particles tend to stay in the forward region and escape detection. Therefore, similar to the single Higgs production, we will consider the inclusive channel in Eq. (17), which is populated dominantly by the

TABLE IV. Predicted cross sections of the inclusive $\mu^{+} \mu^{-} \rightarrow$ $H H+X$, as given in Eq. (18) at different muon collider energies.

\begin{tabular}{lcccccc}
\hline \hline$\sqrt{s}[\mathrm{TeV}]$ & $\sigma_{\mathrm{SM}}[\mathrm{fb}]$ & $R_{1}$ & $R_{2}$ & $R_{3}$ & $R_{4}$ & $R_{5}$ \\
\hline $3 \mathrm{TeV}$ & 0.91 & -3.5 & -0.65 & 3.1 & 14 & 0.49 \\
$6 \mathrm{TeV}$ & 2.0 & -3.9 & -0.50 & 2.8 & 29 & 0.35 \\
$10 \mathrm{TeV}$ & 3.6 & -4.3 & -0.43 & 2.7 & 54 & 0.29 \\
$14 \mathrm{TeV}$ & 4.9 & -4.4 & -0.38 & 2.6 & 80 & 0.25 \\
$30 \mathrm{TeV}$ & 7.6 & -4.4 & -0.28 & 2.3 & 210 & 0.19 \\
\hline \hline
\end{tabular}




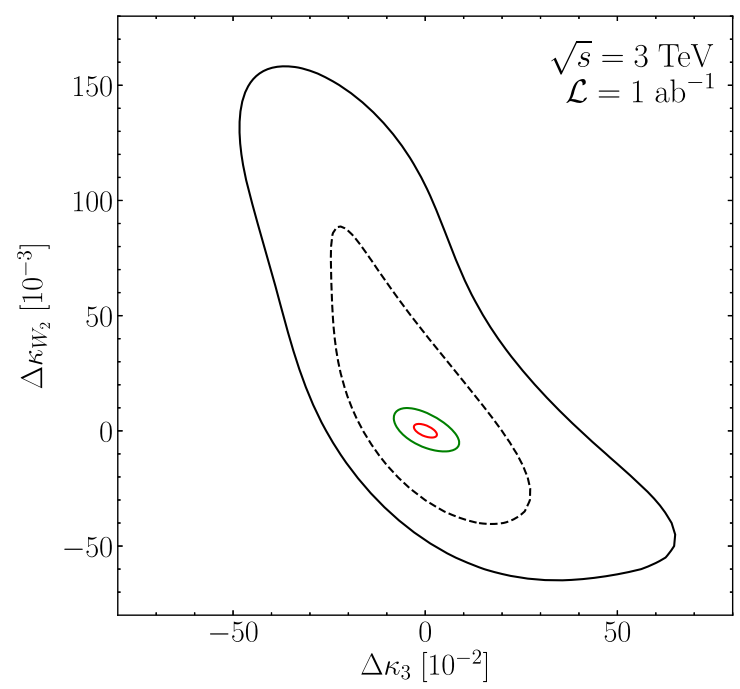

(a)

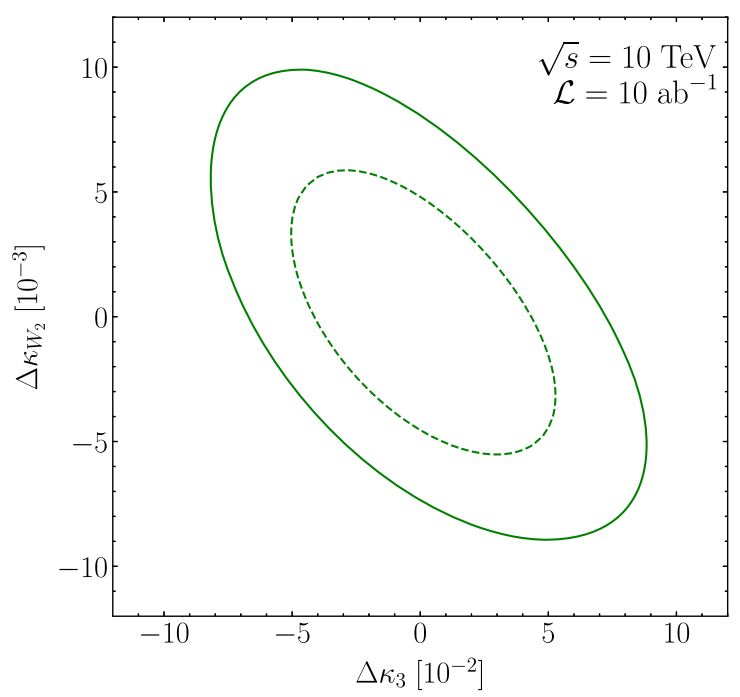

(c)

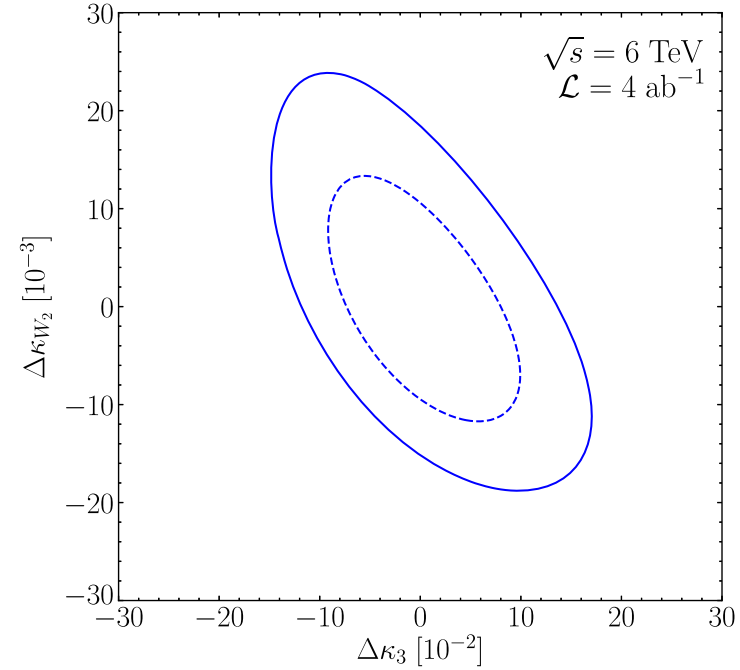

(b)

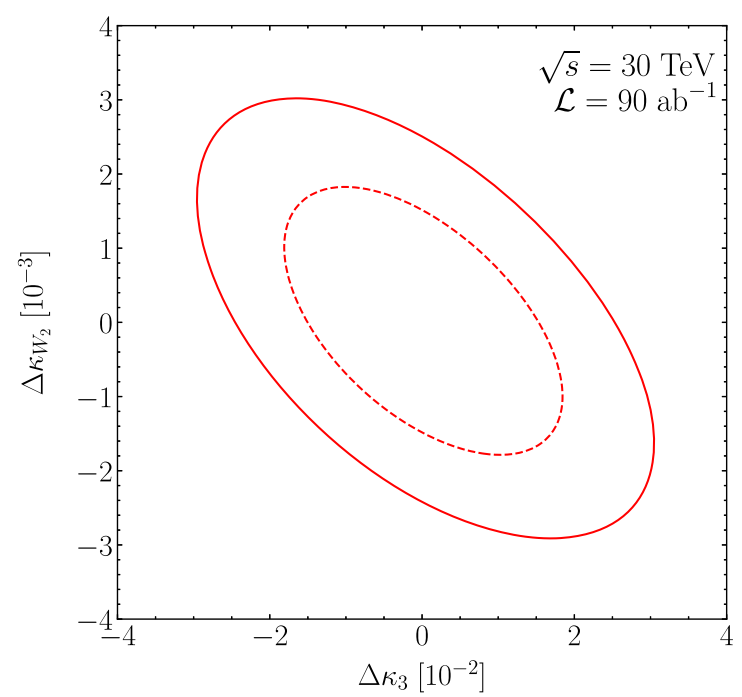

(d)

FIG. 7. Correlated bounds with $95 \%$ C.L. (solid) and $68 \%$ C.L. (dashed) in the $\Delta \kappa_{W_{2}}-\Delta \kappa_{3}$ plane for $\sqrt{s}=3,6$, 10, 30 TeV, respectively. In (a), inner ellipses (solid) include the 95\% C.L. results for $10 \mathrm{TeV}$ and $30 \mathrm{TeV}$ for comparison.

$W W$ fusion and, to a less extent, by the $Z Z$ fusion events when the outgoing muons are too forward to be detected.

The cross section for the inclusive $\mu^{+} \mu^{-} \rightarrow H H+X$ can be parametrized as [38]

$$
\begin{aligned}
\sigma= & \sigma_{\mathrm{SM}}\left[1+R_{1} \Delta \kappa_{W_{2}}+R_{2} \Delta \kappa_{3}+R_{3} \Delta \kappa_{W_{2}} \Delta \kappa_{3}\right. \\
& \left.+R_{4}\left(\Delta \kappa_{W_{2}}\right)^{2}+R_{5}\left(\Delta \kappa_{3}\right)^{2}\right],
\end{aligned}
$$

where the $\sigma_{\mathrm{SM}}$ is the SM cross section. The SM cross section $\sigma_{\mathrm{SM}}$ and coefficients $R_{i}$, before any cuts, are given in Table IV. It is instructive to consider the energy dependence of different classes of Feynman diagrams contributing to $\mathrm{HH}$ production, by studying the partonic scattering $W^{+} W^{-} \rightarrow H H$. As the dominant contribution comes from the longitudinal $W$ scattering $W_{L}^{+} W_{L}^{-} \rightarrow H H$, the scattering amplitude can be written as
TABLE V. Cross sections of the inclusive $\mu^{+} \mu^{-} \rightarrow H H+X \rightarrow$ $b \bar{b} b \bar{b}+X$ in different $m_{H H}$ ranges as the coefficients corresponding to the five terms in Eq. (25) with $\sqrt{s}=10 \mathrm{TeV}$.

\begin{tabular}{lcccccl}
\hline \hline$m_{H H}[\mathrm{GeV}]$ & $\sigma_{\mathrm{SM}}^{\text {cut }}[\mathrm{ab}]$ & $r_{1}$ & $r_{2}$ & $r_{3}$ & $r_{4}$ & $r_{5}$ \\
\hline$[0,350)$ & 15 & -2.7 & -1.7 & 7.6 & 6.7 & 2.6 \\
{$[350,450)$} & 24 & -3.4 & -1.2 & 5.2 & 7.8 & 0.95 \\
{$[450,550)$} & 24 & -4.0 & -0.91 & 4.6 & 12 & 0.52 \\
{$[550,650)$} & 21 & -4.6 & -0.70 & 4.7 & 17 & 0.36 \\
{$[650,750)$} & 17 & -5.3 & -0.60 & 5.1 & 26 & 0.28 \\
{$[750,950)$} & 24 & -6.9 & -0.52 & 6.3 & 46 & 0.23 \\
{$[950,1350)$} & 23 & -11 & -0.47 & 8.7 & 120 & 0.19 \\
{$[1350,5000)$} & 15 & -18 & -0.30 & 7.2 & 240 & 0.075 \\
\hline \hline
\end{tabular}




$$
\mathcal{A}\left(W_{L}^{+} W_{L}^{-} \rightarrow H H\right)=\mathcal{A}_{\mathrm{SM}}+\mathcal{A}_{1} \Delta \kappa_{W_{2}}+\mathcal{A}_{2} \Delta \kappa_{3},
$$

where $\mathcal{A}_{\mathrm{SM}}, \mathcal{A}_{2} \sim$ constant, and $\mathcal{A}_{1} \sim E^{2}$ at high energies $E \gg M_{W}$. Because of the energy growing behavior of $\mathcal{A}_{1}$, the cross section has a strong dependence on $\Delta \kappa_{W_{2}}$ over a large range of phase space. As a result, we expect to be able to constrain $\kappa_{W_{2}}$ better than $\kappa_{3}$. This argument also shows, when extracting the trilinear Higgs self-coupling it is important to consider the impact from the quartic $V V H H$ coupling. In this study, we have assumed the $H H V V$ vertex is modified only in its strength for simplicity, while in many well-motivated new physics models the tensor structure of the quartic coupling could also be corrected $[39,40]$. It will be interesting to further assess the impact of these additional modifications on the extraction of $\kappa_{3}$ [41].

For the Higgs decays, we once again focus on the leading decay channel $H H \rightarrow b \bar{b} b \bar{b}$, which has a SM branching fraction $\operatorname{BR}(4 b) \simeq 34 \%$. We impose basic acceptance cuts

$$
p_{T}(b)>30 \mathrm{GeV}, \quad 10^{\circ}<\theta_{b}<170^{\circ}, \quad \Delta R_{b b}>0.4 .
$$

As before, we further assume the jet energy resolution to be $\Delta E / E=10 \%$.

The Higgs candidates are reconstructed from the four energetic jets. The four jets are paired by minimizing

$$
\left(m_{j_{1} j_{2}}-m_{H}\right)^{2}+\left(m_{j_{3} j_{4}}-m_{H}\right)^{2} .
$$

And for each Higgs candidate, we impose

$$
\left|m_{j j}-m_{H}\right|<15 \mathrm{GeV}
$$

to reject background from $Z$ and $W$ resonances. We also require the recoil mass

$M_{\text {recoil }}=\sqrt{\left(p_{\mu^{+}}+p_{\mu^{-}}-p_{H_{1}}-p_{H_{2}}\right)^{2}}>200 \mathrm{GeV}$.

The signal selection efficiencies and the corresponding cross sections are listed in Table II. If we tighten the angular cut to $20^{\circ}$, the efficiencies would drop by a factor of 3-4.

We again perform a simultaneous fit to $\kappa_{3}$ and $\kappa_{W_{2}}$ using binned maximum likelihood fit. Given the different energy dependence in the subamplitudes controlled by $\kappa_{3}$ and $\kappa_{W_{2}}$, we decided to bin the $m_{H H}$ distribution into the following intervals $^{2}$

$$
m_{H H}=[0,350,450,550,650,750,950,1350,5000] \mathrm{GeV} .
$$

\footnotetext{
${ }^{2}$ A similar procedure for double Higgs production in hadron colliders can be found in Ref. [42].
}

TABLE VI. The accuracies at the $95 \%$ C.L. in $\Delta \kappa_{W_{2}}$ and $\Delta \kappa_{3}$ for the inclusive channel, by varying one coupling at a time.

\begin{tabular}{lccccc}
\hline \hline$\sqrt{s}(\mathrm{TeV})$ & 3 & 6 & 10 & 14 & 30 \\
\cline { 2 - 6 } benchmark lumi $\left(\mathrm{ab}^{-1}\right)$ & 1 & 4 & 10 & 20 & 90 \\
\hline$\left(\Delta \kappa_{W_{2}}\right)_{\text {in }}$ & $5.3 \%$ & $1.3 \%$ & $0.62 \%$ & $0.41 \%$ & $0.20 \%$ \\
$\left(\Delta \kappa_{3}\right)_{\text {in }}$ & $25 \%$ & $10 \%$ & $5.6 \%$ & $3.9 \%$ & $2.0 \%$ \\
\hline \hline
\end{tabular}

The binned cross section of $\mu^{+} \mu^{-} \rightarrow H H+X \rightarrow b \bar{b} b \bar{b}+$ $X$ after the selection cuts can be parametrized, in a similar fashion, as

$$
\begin{aligned}
\sigma^{\mathrm{cut}}= & \sigma_{\mathrm{SM}}^{\mathrm{cut}}\left[1+r_{1} \Delta \kappa_{W_{2}}+r_{2} \Delta \kappa_{3}+r_{3} \Delta \kappa_{W_{2}} \Delta \kappa_{3}\right. \\
& \left.+r_{4}\left(\Delta \kappa_{W_{2}}\right)^{2}+r_{5}\left(\Delta \kappa_{3}\right)^{2}\right],
\end{aligned}
$$

where the values are given in Table $\mathrm{V}$ for $\sqrt{s}=10 \mathrm{TeV}$ for illustration. It is important to note again the increasing sensitivity on $\kappa_{W_{2}}$ at higher values of $m_{H H}$. The resulting contours are shown in Fig. 7. In Table VI we also provide the accuracies at the $95 \%$ C.L. from the single parameter fit, by allowing $\kappa_{3}$ and $\kappa_{W_{2}}$ to vary only one at a time.

\section{DISCUSSION AND CONCLUSION}

As we have shown in this work, a multi-TeV high energy muon collider will have a tremendous potential to constrain the electroweak Higgs couplings with unprecedented accuracy. It will offer a unique probe into the nature of the Higgs boson as well as the scale of possible new physics beyond the SM. In Table VII, we present a summary of the estimated sensitivities at different collider energies and luminosities. In the last column of the table, we compare with the expected precision from other proposed colliders. It is clear that a multi-TeV muon collider could improve the measurements substantially.

It is possible to translate the bound in the $\kappa$-scheme into the constraint on $\Lambda$, the scale of new physics associated with the dim-6 operators in Eq. (2),

$$
\Lambda \sim \sqrt{\frac{c_{H, 6}}{\Delta \kappa}} v
$$

Assuming $c_{6, H} \sim \mathcal{O}(1)$, the scale is estimated to be $\Lambda \sim 1 \mathrm{TeV} / \sqrt{16 \Delta \kappa}$, as shown in Table VII. A summary figure, which combines our results for the coupling measurements, is given in Fig. 8, with the upper horizontal axis marking the estimated scale $\Lambda$ in TeV. With $\Lambda / \sqrt{c}_{i} \sim$ (10-16) $\mathrm{TeV}$ at a collider of (10-30) TeV, we would be probing new physics at very high scales or deeply into quantum effects.

In our analyses, we only focused on the leading decay channel $H \rightarrow b \bar{b}$. A more comprehensive study could include the other decay channels as well, such as $H \rightarrow$ $W W, Z Z, \tau \tau, c \bar{c}$ and $\gamma \gamma, g g$, to further improve the precision. On the other hand, due to the lack of knowledge of the 
TABLE VII. Summary table of the expected accuracies at 95\% C.L. for the Higgs couplings at a variety of muon collider energies and luminosities.

\begin{tabular}{lcccccc}
\hline \hline$\sqrt{s}$ (lumi.) & $3 \mathrm{TeV}\left(1 \mathrm{ab}^{-1}\right)$ & $6(4)$ & $10(10)$ & $14(20)$ & $30(90)$ & Comparison \\
\hline$W W H\left(\Delta \kappa_{W}\right)$ & $0.26 \%$ & $0.12 \%$ & $0.073 \%$ & $0.050 \%$ & $0.023 \%$ & $0.1 \%[43]$ \\
$\Lambda / \sqrt{c_{i}}(\mathrm{TeV})$ & 4.7 & 7.0 & 9.0 & 11 & 16 & $(68 \%$ C.L. $)$ \\
$Z Z H\left(\Delta \kappa_{Z}\right)$ & $1.4 \%$ & $0.89 \%$ & $0.61 \%$ & $0.46 \%$ & $0.21 \%$ & $0.13 \%[17]$ \\
$\Lambda / \sqrt{c}_{i}(\mathrm{TeV})$ & 2.1 & 2.6 & 3.2 & 3.6 & 5.3 & $(95 \%$ C.L. $)$ \\
$W W H H\left(\Delta \kappa_{W_{2}}\right)$ & $5.3 \%$ & $1.3 \%$ & $0.62 \%$ & $0.41 \%$ & $0.20 \%$ & $5 \%[38], 1 \%[24]$ \\
$\Lambda / \sqrt{c}_{i}(\mathrm{TeV})$ & 1.1 & 2.1 & 3.1 & 3.8 & 5.5 & $(68 \%$ C.L. $)$ \\
$H H H\left(\Delta \kappa_{3}\right)$ & $25 \%$ & $10 \%$ & $5.6 \%$ & $3.9 \%$ & $2.0 \%$ & $5 \%[22,23]$ \\
$\Lambda / \sqrt{c}_{i}(\mathrm{TeV})$ & 0.49 & 0.77 & 1.0 & 1.2 & 1.7 & $(68 \%$ C.L. $)$ \\
\hline \hline
\end{tabular}

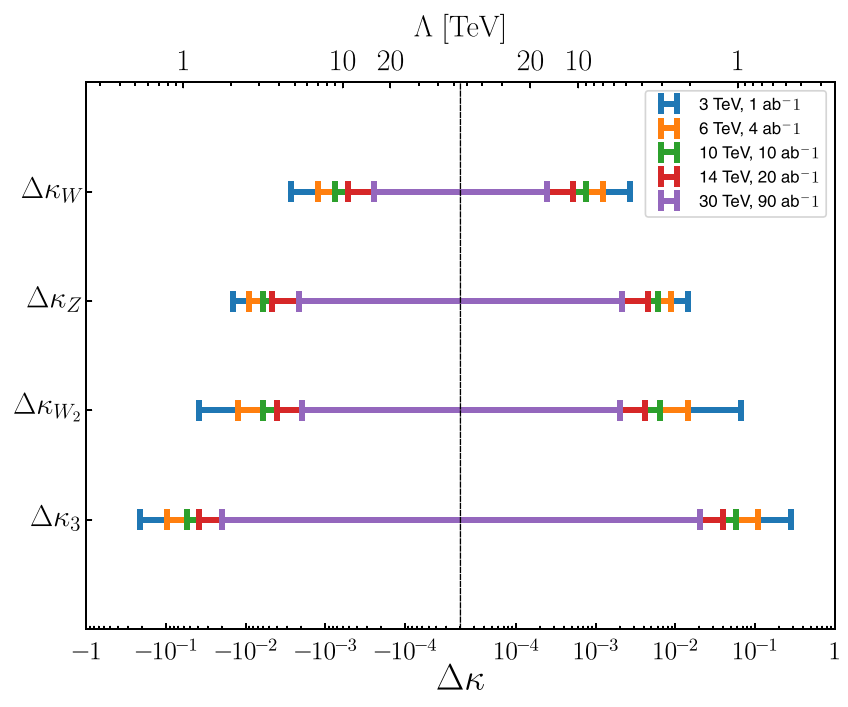

FIG. 8. Summary of the expected accuracies at 95\% C.L. for the Higgs couplings at a variety of muon collider energies and luminosities. The upper horizontal axis marks the accessible scale $\Lambda$, assuming $c_{6, H} \sim \mathcal{O}(1)$.

specifics of the detector design, we have not made any attempts for experimental detector simulations. Further work may be needed to draw a more complete conclusion for the expected sensitivity reach.

In summary, we estimated the expected precision at a multi-TeV muon collider for measuring the Higgs boson couplings with electroweak gauge bosons, $H V V$ and $H H V V$, as well as the trilinear Higgs self-coupling $\mathrm{HHH}$. With the anticipated high $\mathrm{CM}$ energies and high luminosities, a multi- $\mathrm{TeV}$ muon collider could provide us with unparalleled precision for Higgs physics and, consequently, offer some of the most stringent experimental tests of the SM Higgs sector. As we have shown in this study, the outgoing remnant particles have a strong tendency to stay in the very forward region. The enhanced collinear behavior of the final state particles results in the dominant configuration of "inclusive" processes, a notion usually reserved for hadron colliders, unless there is a device to detect the very forward muons of a few degrees from the beam. These features add new subtlety to Higgs coupling measurements, since it is now difficult to isolate $W W$ fusion from $Z Z$ fusion events in the Higgs production. We addressed the subtlety by performing binned maximum likelihood analyses to simultaneously fit two parameters involved in the inclusive processes. The approach and methodology adopted in this study could be applicable to new physics searches at a high energy muon collider.

\section{ACKNOWLEDGMENTS}

The work of T.H. was supported in part by the U.S. Department of Energy under Grant No. DE-FG0295ER40896 and in part by the PITT PACC. The work of D. L. was supported in part by the U.S. Department of Energy under Grant No. DE-SC-0009999. I. L. is supported in part by the U.S. Department of Energy under Contracts No. DE- AC02-06CH11357 at Argonne and No. DESC0010143 at Northwestern. X. W. was supported by the National Science Foundation under Grant No. PHY1915147.
[1] P. Sikivie, L. Susskind, M. B. Voloshin, and V. I. Zakharov, Isospin breaking in technicolor models, Nucl. Phys. B173, 189 (1980).
[2] S. Schael et al. (ALEPH, DELPHI, L3, OPAL, SLD, LEP Electroweak Working Group, SLD Electroweak Group, SLD Heavy Flavour Group Collaboration), Precision 
electroweak measurements on the $Z$ resonance, Phys. Rep. 427, 257 (2006).

[3] B. Grzadkowski, M. Iskrzynski, M. Misiak, and J. Rosiek, Dimension-six terms in the standard model lagrangian, J. High Energy Phys. 10 (2010) 085.

[4] V. Barger, T. Han, P. Langacker, B. McElrath, and P. Zerwas, Effects of genuine dimension-six Higgs operators, Phys. Rev. D 67, 115001 (2003).

[5] G. Giudice, C. Grojean, A. Pomarol, and R. Rattazzi, The strongly-interacting light Higgs, J. High Energy Phys. 06 (2007) 045.

[6] I. Low, R. Rattazzi, and A. Vichi, Theoretical constraints on the Higgs effective couplings, J. High Energy Phys. 04 (2010) 126.

[7] X. Zhang, Operator analysis for the Higgs potential and cosmological bound on the Higgs-boson mass, Phys. Rev. D 47, 3065 (1993).

[8] C. Grojean, G. Servant, and J. D. Wells, First-order electroweak phase transition in the standard model with a low cutoff, Phys. Rev. D 71, 036001 (2005).

[9] X. Gan, A. J. Long, and L.-T. Wang, Electroweak sphaleron with dimension-six operators, Phys. Rev. D 96, 115018 (2017).

[10] G. Aad et al. (ATLAS Collaboration), Combined measurements of Higgs boson production and decay using up to $80 \mathrm{fb}^{-1}$ of proton-proton collision data at $\sqrt{s}=13 \mathrm{TeV}$ collected with the ATLAS experiment, Phys. Rev. D 101, 012002 (2020).

[11] A. M. Sirunyan et al. (CMS Collaboration), Combined measurements of Higgs boson couplings in protonproton collisions at $\sqrt{s}=13 \mathrm{TeV}$, Eur. Phys. J. C 79, 421 (2019).

[12] M. Cepeda et al., Report from Working Group 2: Higgs physics at the HL-LHC and HE-LHC, CERN Yellow Rep. Monogr. 7, 221 (2019).

[13] D. Asner et al., ILC Higgs white paper, arXiv:1310.0763.

[14] J. Tian and K. Fujii (ILD Collaboration), Measurement of Higgs couplings and self-coupling at the ILC, Proc. Sci., EPS-HEP2013 (2013) 316 [arXiv:1311.6528].

[15] A. Abada et al. (FCC Collaboration), FCC-ee: The lepton collider: Future circular collider conceptual design report volume 2, Eur. Phys. J. Special Topics 228, 261 (2019).

[16] M. Dong et al. (CEPC Study Group Collaboration), CEPC conceptual design report: Volume 2-Physics \& detector, arXiv:1811.10545.

[17] F. An, Y. Bai, C. Chen, X. Chen, Z. Chen, J. G. da Costa, Z. Cui, Y. Fang, C. Fu, J. Gao et al., Precision Higgs physics at the CEPC, Chin. Phys. C 43, 043002 (2019).

[18] A. Robson and P. Roloff, Updated CLIC luminosity staging baseline and Higgs coupling prospects, arXiv:1812.01644.

[19] P. Roloff, U. Schnoor, R. Simoniello, and B. Xu (CLICdp Collaboration), Double Higgs boson production and Higgs self-coupling extraction at CLIC, Eur. Phys. J. C 80, 1010 (2020).

[20] V. D. Barger and T. Han, Double Higgs boson production via $W W$ fusion in Tev $e^{+} e^{-}$collisions, Mod. Phys. Lett. A 05, 667 (1990).
[21] W. Kilian, S. Sun, Q.-S. Yan, X. Zhao, and Z. Zhao, MultiHiggs boson production and unitarity in vector-boson fusion at future hadron colliders, Phys. Rev. D 101, 076012 (2020).

[22] A. Abada et al. (FCC Collaboration), FCC-hh: The hadron collider: Future circular collider conceptual design report volume 3, Eur. Phys. J. Special Topics 228, 755 (2019).

[23] M. Ahmad et al., CEPC-SPPC preliminary conceptual design report. 1. Physics and detector, Technical Report, 3, 2015.

[24] F. Bishara, R. Contino, and J. Rojo, Higgs pair production in vector-boson fusion at the LHC and beyond, Eur. Phys. J. C 77, 481 (2017).

[25] M. Chiesa, F. Maltoni, L. Mantani, B. Mele, F. Piccinini, and X. Zhao, Measuring the quartic Higgs self-coupling at a multi-TeV muon collider, J. High Energy Phys. 09 (2020) 098.

[26] S. Gori and I. Low, Precision Higgs measurements: Constraints from new oblique corrections, J. High Energy Phys. 09 (2013) 151.

[27] J. P. Delahaye, M. Diemoz, K. Long, B. Mansoulié, N. Pastrone, L. Rivkin, D. Schulte, A. Skrinsky, and A. Wulzer, Muon colliders, arXiv:1901.06150.

[28] K. Long, D. Lucchesi, M. Palmer, N. Pastrone, D. Schulte, and V. Shiltsev, Muon colliders: Opening new horizons for particle physics, arXiv:2007.15684.

[29] D. Buttazzo, D. Redigolo, F. Sala, and A. Tesi, Fusing vectors into scalars at high energy lepton colliders, J. High Energy Phys. 11 (2018) 144.

[30] A. Costantini, F. De Lillo, F. Maltoni, L. Mantani, O. Mattelaer, R. Ruiz, and X. Zhao, Vector boson fusion at multi-TeV muon colliders, J. High Energy Phys. 09 (2020) 080 .

[31] T. Han, Y. Ma, and K. Xie, High energy leptonic collisions and electroweak parton distribution functions, arXiv:2007.14300.

[32] R. Capdevilla, D. Curtin, Y. Kahn, and G. Krnjaic, A guaranteed discovery at future muon colliders, arXiv: 2006.16277.

[33] V. D. Barger, M. Berger, J. Gunion, and T. Han, $s$ Channel Higgs Boson Production at a Muon Muon Collider, Phys. Rev. Lett. 75, 1462 (1995).

[34] V. D. Barger, M. Berger, J. Gunion, and T. Han, Higgs Boson physics in the s channel at $\mu^{+} \mu^{-}$colliders, Phys. Rep. 286, 1 (1997).

[35] J. Alwall, R. Frederix, S. Frixione, V. Hirschi, F. Maltoni, O. Mattelaer, H. S. Shao, T. Stelzer, P. Torrielli, and M. Zaro, The automated computation of tree-level and next-toleading order differential cross sections, and their matching to parton shower simulations, J. High Energy Phys. 07 (2014) 079.

[36] T. Han, Z. Liu, Z. Qian, and J. Sayre, Improving Higgs coupling measurements through $\mathrm{ZZ}$ fusion at the ILC, Phys. Rev. D 91, 113007 (2015).

[37] M. Selvaggi, Proceedings of the mdi studies meeting of the muon collider collaboration, https://indico.cern.ch/ event/957299/contributions/4023467/attachments/2106044/ 3541874/delphes_card_mucol_mdi\%20.pdf (2020).

[38] R. Contino, C. Grojean, D. Pappadopulo, R. Rattazzi, and A. Thamm, Strong Higgs interactions at a linear collider, J. High Energy Phys. 02 (2014) 006. 
[39] D. Liu, I. Low, and Z. Yin, Universal Imprints of a PseudoNambu-Goldstone Higgs Boson, Phys. Rev. Lett. 121, 261802 (2018).

[40] D. Liu, I. Low, and Z. Yin, Universal relations in composite Higgs models, J. High Energy Phys. 05 (2019) 170.
[41] T. Han, D. Liu, I. Low, and X. Wang (to be published).

[42] C.-R. Chen and I. Low, Double take on new physics in double Higgs boson production, Phys. Rev. D 90, 013018 (2014).

[43] R. Franceschini et al., The CLIC potential for new physics, CERN Yellow Rep. Monogr. 3 (2018). 